each set of cutpoints. Measures of effect were weaker using the manufacturer's cutpoints compared to the modified cutpoints. Age was not significantly correlated with positive vs. negative serostatus using the manufacturer's cutpoint (relative risk ratio $(\mathrm{RRR})=1.03,95 \% \mathrm{CI}$ : 0.97-1.09), but the association strengthened using the modified cutpoint $(R R R=1.09$, 95\% CI: 1.01-1.17). Using the manufacturer's cutpoint, the RRR for BV was 1.73 (95\% CI: $0.71-4.19$ ) vs. $R R R=2.70$ (95\% CI: 0.94-7.79) for the modified cutpoint. For partner concurrency, the RRR using the manufacturer's cutpoint was 1.47 (95\% CI: $0.71-3.07$ ), vs. $\mathrm{RRR}=3.45$ (95\% CI: $1.12-$ 10.57) for the modified threshold.

Conclusion Changing the test cutpoints affected observed associations with previously-identified HSV-2 risk factors. HSV-2 prevention interventions informed by correlates of HSV-2 infection must be aware that different target populations may emerge depending on which cutpoints are adopted.

Disclosure No significant relationships.

\section{P095 AGE DISPARITY AND SOCIODEMOGRAPHIC CORRELATES OF HERPES SIMPLEX VIRUS TYPE 2 (HSV- 2) SEROPOSITIVITY IN SOUTH INDIA}

${ }^{1}$ Makella Coudray* ${ }^{2}$ Abraham Degarege, ${ }^{3}$ Anisa Khan, ${ }^{3}$ Kavitha Ravi, ${ }^{3}$ Vijaya Srinivas, ${ }^{4}$ Jeffrey Klausner, ${ }^{2}$ Purnima Madhivanan, ${ }^{5}$ Caitlyn Placek. ${ }^{1}$ Florida International University, Epidemiology, Miami, USA; ${ }^{2}$ Florida International University, Epidemiology, Miami, USA; ${ }^{3}$ Public Health Research Institute of India, Mysore, India; ${ }^{4}$ UCLA David Geffen School of Medicine and Fielding School of Public Health, Epidemiology, Los Angeles, USA; ${ }^{5}$ Ball State University, Epidemiology, Muncie, USA

\subsection{6/sextrans-2019-sti.290}

Background Age, gender, sexual history, marital status, education, socioeconomic status and geographic location are known risk factors of Herpes simplex virus type 2 (HSV-2) seroprevalence. There is a paucity of data on the potential association between increasing age difference between sex partners and HSV-2 infection. This study investigated the relationship between increasing age difference between sex partners and HSV-2 infection in Mysore, India, due to a lack of studies documenting this phenomenon in HSV-2 seropositive individuals in India.

Methods This study presents one section of a larger comparative study conducted between October 2016 and July 2017 in collaboration with the Public Health Research Institute of India, that assessed drug use and risky sexual behavior among adults in Mysore, India. Study personnel conducted structured interviews with men and women to assess demographic variables, reproductive health, risky sexual behavior and relationship history. ELISA testing (Focus Diagnostics HerpeSelect ${ }^{\circledR} 2$ ELISA IgG, Focus Technologies, Cypress, CA) was used to determine the presence of HSV-2 antibodies.

Results There were 351 participants included in the study. The prevalence of HSV-2 in this sample was 9.4\% (95\%CI: $6.3 \%, 12.5 \%)$. HSV-2 infection was correlated with partner age difference and religio. The odds of HSV-2 infection significantly increased among the study participants with an increase in the age difference with their sex partner (OR: 1.22, 95\% CI: $1.06,1.40)$. The odds of HSV-2 infection was significantly lower among Hindus as compared to non-Hindus (OR: 0.19, 95\% CI: $0.04,0.84)$. There was no significant difference in the prevalence of HSV-2 infection by sex, education, or occupation.

Conclusion HSV-2 seroprevalence was relatively low in this population compared to regional and global rates. HSV-2 seropositivity was associated with an increasing partner age difference. This highlights the need for further research in this area in different Indian settings to determine what the dynamics of this phenomenon.

Disclosure No significant relationships.

\section{P096 VULVAR ZOSTER}

${ }^{1}$ Christine Remar Concepcion*, ${ }^{2}$ Maria Bandola. ${ }^{1}$ University of the Philippines, Department of Obstetrics and Gynecology-Section of Infectious Diseases, Manila City, Philippines; ${ }^{2}$ University of the Philippines - Philippine General Hospital, Manila, Philippines

10.1136/sextrans-2019-sti.291

Background Herpes Zoster infection is part of the two distinct syndromes of infections secondary to varicella-zoster. The other syndrome being the primary infection of varicella (chickenpox) which is a contagious but benign illness. Reactivation of this primary infection results to herpes zoster or shingles. Shingles is a uniquely localized disease with unilateral radicular pain and a vesicular rash limited to the area of the skin innervated by a single dorsal root or cranial sensory ganglion. Clinically significant pain usually accompanies the dermatomal rash of herpes zoster. This pain may persist for weeks, months, or even years after the herpes zoster rash has healed, a debilitating complication known as (PHN) postherpetic neuralgia. This condition disables a individuals infected with shingles especially among the elderly. We are presented with a rare case of vulvar zoster in a 52 year old nulligravid. The case presented with pain, pruritus, rashes and eventually ulcers along the dermatomal line of the vulva. Clinical improvement was noted upon treatment with an anti-viral.

Disclosure No significant relationships.

\section{P099 RISK OF ACQUIRING HIV INFECTION AMONG EXPOSED INFANTS IN SELECTED PRIMARY HEALTH CARE FACILITIES IN IBADAN OYO STATE NIGERIA}

${ }^{1}$ Aishat Usman*, ${ }^{2}$ Olubunmi Ayinde, ${ }^{3}$ Esther Asekun-Olarinmoye. ${ }^{1}$ Texila American University, Public Health, Georgetown, Guyana; ${ }^{2}$ Oyo State Ministry of Health, STI and HIV, Ibadan, Nigeria; ${ }^{3}$ Osun State University, Community Medicine, Osogbo, Nigeria

\subsection{6/sextrans-2019-sti.292}

Background In 2016, Nigeria recorded 37,000 new HIV infections among children out of the total of 60,000 new infections in West and Central Africa, representing 62 per cent of the new infections. This study was conducted to determine the outcomes of HIV exposed infants and the risk of acquiring HIV infections at the final outcome of 18 months

Methods This is a retrospective cohort study of exposed infants between January 2015-december 2016.A proforma was used to abstract information from clinic records on socio-demographics characteristics, ARV prophylaxis, mode of delivery, birth weight, infant prophylaxis, Early infant diagnosis (EID), referral to ART. Cox proportion hazard regression model was 